\title{
Increased Tissue Pressure Induces Demyelinated Lesions and Necroses in the Brain
}

\author{
${ }^{1}$ Helmut Barz, ${ }^{2}$ Ulrich Barz and ${ }^{3}$ Almut Schreiber \\ ${ }^{1}$ Department of Neuropathology, Dietrich-Bonhoeffer Hospital Neubrandenburg, Mecklenburg-West-Pomerania, Germany \\ ${ }^{2}$ Martin-Luther-Universität Halle-Wittenberg, Halle, Saxony-Anhalt, Germany \\ ${ }^{3}$ Consultant Occupational Health Physician, Institut Für Arbeits-und Sozialhygiene, Aktiengesellschaft, \\ Dresden, Saxony, Germany
}

Article history

Received: 08-12-2014

Revised: 20-01-2015

Accepted: 12-03-2015

Corresponding Author:

Helmut Barz

Department of Neuropathology,

Dietrich-Bonhoeffer Hospital

Neubrandenburg, Mecklenburg-

West-Pomerania, Germany

Email: helmutbarz@gmx.de

\begin{abstract}
A general brain edema increases the size of the brain tissue and stretches the elastic neuronal and glial fibers. Myelinated fibers are more elastic than unmyelinated fibers. The stretching of the neuronal fibers leads particular in regions of the white matter or areas surrounded by myelinated fiber tracts to an increased tissue pressure. This physical principle can be the reason for demyelination and tissue necrosis. The higher the tissue pressure, the lower the blood perfusion. We support the pressure-hypothesis by comparison with well-known pressure related pathologies of the nervous system and other organs. One convincing example is the central pontine myelinolysis. A general or local tissue edema may lead especially in the periventricular and the deep white matter, but also in the cerebellum, the brain stem and the spinal cord to the formation of hypoxemic lesions. In consequence, this could result in diseases like leukoaraiosis, Binswanger encephalopathy, idiopathic intracranial hypertension and Multiple Sclerosis (MS). In cases of brain tissue swelling, capsula-like structures may lead to a rapid increase in tissue pressure within the enclosed areas. This may occur particularly at the optic nerve. A reduced reabsorption of Cerebrospinal Fluid (CSF) can also lead to an increase of the pressure in the Central Nervous System (CNS).
\end{abstract}

Keywords: Brain Edema, Tissue Elasticity, Tissue Pressure, White Matter Diseases, Central Pontine Myelinolysis, Multiple Sclerosis

\section{Introduction}

A particular aspect of this paper is the demonstration of the close connection between tissue edema and a consecutive increase in tissue pressure. The blood supply changes in direct relationship with this physical principle. Pressure induced ischemia is well-known in many organs. Decubitus ulcers are a well-known example. Another physical principle is edematous swollen tissue in a limited space, e.g., necrosis of the tubuli contorti of the testis after trauma, the compartment syndrome (necroses of the skeletal muscles), or the brain death syndrome. In these cases the edematous injured tissue is prevented from expanding in volume by the surrounding capsula-like structures (tunica albuginea, fasciae, cranium). The tissue pressure may reach the capillary blood pressure and the blood supply drops to zero.

Although scientists endeavor to understand the pathogenesis of demyelinating diseases, many questions remain unresolved. An example is the pathogenesis of MS. During recent years, the apparently re-discovered venous vessels in the center of the MS plaques have attracted the attention of scientific journals and media. It has been assumed that there are stenoses or other obstructive changes in the great venous vessels or sinuses of the brain. Venous changes could reduce the circulatory bloodstream as a result of a chronic cerebrospinal venous insufficiency and induce the formation of MS plaques (Zamboni et al., 2009). However, such venous stenosis or other changes causing narrowing of blood vessels can hardly be proven (Valdueza et al., 2013). Nevertheless, the regular demonstration of venules in the center of the MS lesions offer a valuable link for the understanding of the mechanism for the formation of the lesions and point to an ischemic origin (Beggs, 2013).

Locally elevated focal tissue pressure could explain the formation of ischemic lesions in gray and white 
matter. Although it seems plausible that an increased local pressure may cause demyelinated lesions, this physical connection has not yet been sufficiently described in the scientific literature.

\section{Demyelinating Diseases}

\section{Etiology of Demyelinating Diseases}

Many demyelinating diseases have a genetic origin and can be summarized as leukodystrophien. The etiology of other demyelinating diseases is unknown. Included here is leukoaraiosis, Binswanger encephalopathy, idiopathic intracranial hypertension and MS. An infective cause of these diseases has not been identified. An inflammatory process caused by autoimmune reactions has been discussed, but no evidence has been provided for this hypothesis (Juurlink, 2013).

MS is not considered to be a hereditary disease. However, the probability of developing MS is about 10 times higher in relatives of patients than in the general population. Women are affected by MS two to three times as often as men, but in people over forty primary and secondary progressive MS affects males and females in roughly equal numbers (Miller and Leary, 2007).

\section{Macroscopic and Microscopic Findings in Demyelinated Lesions}

The MS lesions affect most commonly the white matter in the optic nerve and spinal cord, as well as the brain stem, the cerebellum and the white matter tracts close to the brain ventricles. Since the gray matter lacks a substantial number of myelin sheaths, demyelinated lesions will not be conspicuous in the cerebral cortex. In MS plaques the myelin sheaths of the neuronal fibers are damaged and a great number of axons and oligodendroglial cells are destroyed. In early lesions macrophages, fiberforming astrocytes and a variable degree of perivascular inflammatory infiltrate can be found. Older lesions are transformed into gliotic scars. All the findings are compatible with circumscribed ischemic lesions.

\section{Brain Edema and Brain Tissue Pressure}

Walls of the skull, the spinal column and the dura mater enclose the CNS. In cases of increase of the brain tissue volume (e.g., by mild edema) the intracranial pressure does not change substantially as Cerebrospinal Fluid (CSF) and blood can leave the enclosed space (Monro-Kellie doctrine). Nevertheless, the pressure within the brain increases because the brain tissue does not behave like a fluid.

Brain tissue has viscoelastic respective poroelastic properties (Miller and Adams 1992; Konofagou et al., 2001). Edema or cellular swelling of the whole brain leads to unequal tissue pressures, i.e., pressure gradients in different regions of the brain (Baust et al., 1963).
Crucial to the blood flow is the perfusion pressure, which is the difference between the mean blood pressure and the local tissue pressure. In tissue with reduced blood circulation, hypoxemia will be greater at the venous than at the arterial side of the vascular network (Beggs, 2013). This fact may illustrate the regular occurrence of venous vessels in the center of MS plaques.

\section{Different Tissue Structure as Reason for Different Tissue Pressure in Cases of Brain Edema}

The white matter has a more solid consistency and a greater pressure-elasticity than the cerebral cortex or the basal ganglia (Baust et al., 1963; Krug and Sandig, 1965). Young's modulus, also known as the tensile or elastic modulus, is a measure of the stiffness of an elastic material. Young's modulus (which used an indentation test apparatus) is about $1800 \mathrm{~Pa}$ for white matter and about $1200 \mathrm{~Pa}$ for gray matter (Kaster et al., 2011). However, depending on the method and the location of the examination there are different results (Kaster et al., 2011). Neuritic fibers are comparable to elastic rubber bands (Van Essen, 1997). Swelling of the brain leads to pressure gradients especially at borderline areas of gray and white matter.

\section{Reduced Cerebral Blood Flow in Multiple Sclerosis Patients}

It has been shown that the absolute cerebral blood flow (Law et al., 2004) and the volume of blood in the vessels (Ge et al., 2009; Zivadinov et al., 2011), especially in the white matter and subcortical gray matter, is reduced in MS patients. Law et al. (2004) identified a $52 \%$ decrease in cerebral blood flow throughout the normal-appearing white matter in patients with relapsing-remitting MS compared with controls. Ge et al. (2009) and Zivadinov et al. (2011) reported a marked reduction in venous vasculature visibility on susceptibility-weighted imaging compared with controls. The mean transit time in the blood vessels is increased (Mancini et al., 2012). Juurlink (2013) writes that hypoxia is a precipitating factor in causing early MS lesions and that hypoperfusion may be due to a decrease in an arterial supply, restricted venous return, or a combination of these. Beggs (2013) suggests that other pathophysiological mechanisms must be at work, which are increasing the hydraulic resistance of the cerebral vascular bed in patients with MS and leukoaraiosis. No consideration was given to a possible increased tissue pressure (e.g., by edema) as a cause for the reduction of blood flow and for hypoxemic lesions.

\section{Central Pontine Myelinolysis (CPM), (Osmotic Myelinolysis)}

Tissue regions surrounded by tracts or cords of myelinated fibers are especially jeopardized by edema or 
pressure forces, respectively. A classic example of pressure induced necrosis is the CPM. This often fatal disease (paralysis, "locked in syndrome") is primarily initiated by an electrolyte disturbance with edematous tissue swelling, especially at the center of the pons. Extrapontine lesions (EPM) are found most symmetrically at the corpus geniculatum laterale, the capsula externa, the thalami, the basal ganglia, the cortex-white matter junction and the spinal cord (Chua et al., 2002; Tatewaki et al., 2012). Chua et al. (2002) have described the importance of the "graywhite apposition" for the cortical location of demyelinated lesions.

Messert et al. (1979) wrote about the pathogenesis of the CPM and stated "predilection for the basis pontis could be due to the "grid" effect, especially by the transverse fibers (pedunculi cerebellaris medius) that form (together with the descending tracts) the characteristic grid of the ventral pons. Edema of the grid would literally strangle the myelin sheaths and the small blood vessels. More severe edema would lead to demyelination, necrosis and death." The elevated pressure may develop in particular due to the distension of the broad elastic myelinated fiber tracts in the outer zone of the pons (Fig. 1).

The pressure within the tissue is higher in the center of such a stretched area than at the rim of it. This may also be compared with an "onion bulb" phenomenon, with the core in the central basis pontis and layers extending out with a tapering above and below (Messert et al., 1979). This shows that the highest pressure develops in the midmost layer of a (swollen) onion bulb or the edematous swollen basis pontis, respectively (Fig. 2).

A similar phenomenon may cause demyelination and necrosis in the spinal cord. The edematous swollen gray matter is located in the center and the long myelinated fiber tracts lay at the outer zone of the myelon.

The edematous pressure effect on the pons and on the symmetrical extrapontine locations has been largely neglected for the benefit of an "osmotic demyelination syndrome" (Martin, 2004). The electrolyte disturbance and the rapid correction of hyponatremia recognized as causes of CPM leads without doubt to a general cytotoxic edema. However, the edema provokes demyelination predominantly in specific symmetrical areas of the brain, which can only be explained by the "grid" effect, respectively the "onion bulb" phenomenon.

Chua et al. (2002) stated a similarity between osmotic myelinolysis and lesions in MS. The authors recommend the advanced functional magnetic resonance sequence of diffusion-weighted imaging with apparent diffusion coefficient measurement for the radiologic diagnostic of both diseases because of its inherent sensitivity to water diffusion.

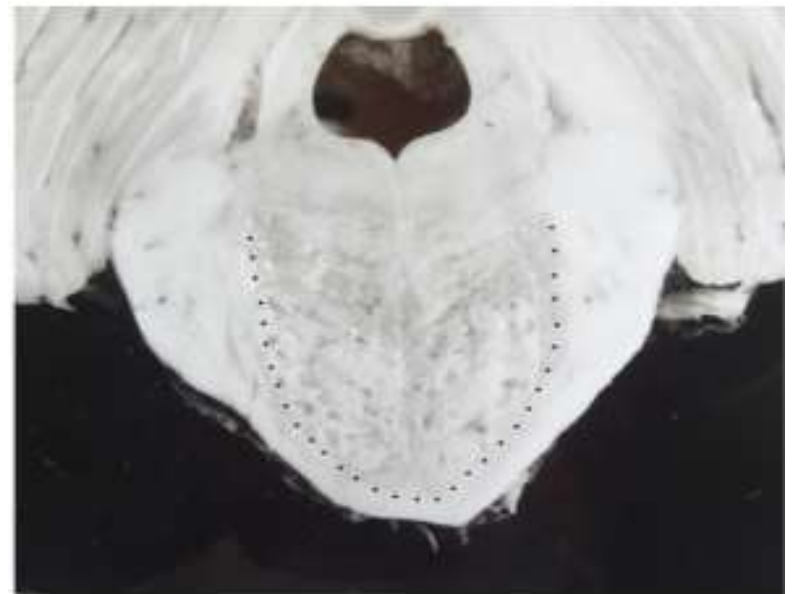

Fig. 1. Central pontine myelinolysis. Necrosis of the basis pontis. See the well preserved myelinated tracts at the outer zone of the basis pontis

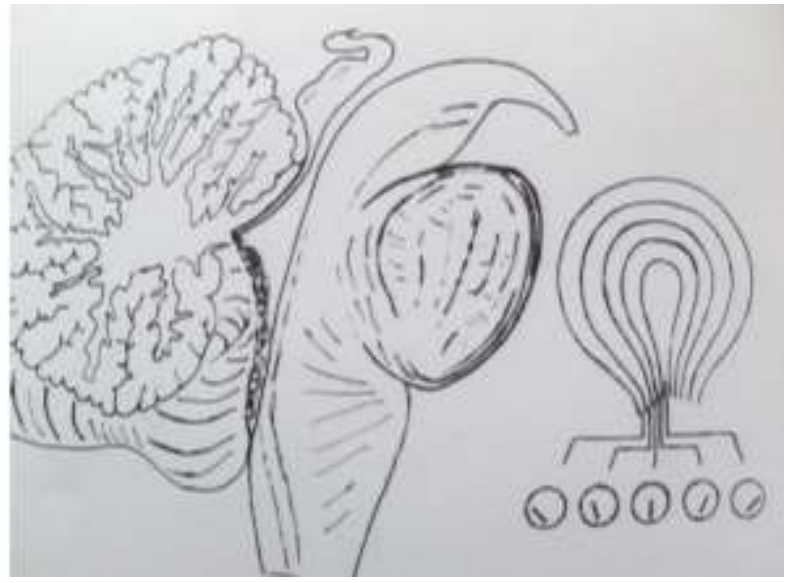

Fig. 2. Schematic demonstration of the onion bulb phenomenon at the pons cerebri. The hydrostatic tissue pressure is the greatest at the center of stretched, overlapping elastic lamellae

\section{Other Examples of Pressure Induced Tissue Necroses in the Nervous System}

A pressure-induced rim of edema and a demyelinated tissue zone surrounds rapidly growing tumors or expanding cancer metastases in the white matter. Tumor necroses can regularly be seen in the center of such neoplasms.

The appearance of symmetrical necrosis in the globus pallidus after survival of carbon monoxide poisoning, cardiac arrest, or hypoglycemia is well known (Graham, 1992). Such necrosis is likely to be caused by a relatively high tissue swelling in the globus pallidus due to edema and an elevated tissue pressure due to distension of the surrounded tissue since this tissue is richer in myelinated fibers.

Patients with long standing epilepsy show necrosis of neuronal cells at the CA1 region in the twisted area of 
the hippocampus (German "Ammonshorn"). The ischemic lesion may be caused by tissue compression within this special anatomical structure.

Necrosis of nerve cells in general hypoglycemia or hypoxia is not distributed diffusely or selected at random. The hypoxemic lesions are accentuated in certain regions, similar to the lesions of the EPM. A characteristic distribution pattern of hypoxemic neuronal cell necrosis is seen in Fig. 3. Damage of nerve cells in the cortex is commonly greater in the depths and at the margins of sulci than at the crest of gyri. The deep-lying gyri of the insula are more affected than other gyri.

It is highly likely that hypoxemic lesions in those regions occur due to structural characteristics and pressure gradients in the edematous swollen tissue.

In cases of supra-or infratentorial expanding brain lesions, pressure differences in both intracranial compartments may occur with hemorrhagic or ischemic pressure necrosis of the herniated brain tissue at the edge of the tentorium.

The "brain death" syndrome is the result of a high tissue pressure of the whole brain in the skull often induced by edema due to intoxication, severe hypoxemia, or trauma.

Peripheral nerves also show long-term damage in cases of acute strains. The blood flow is reduced by $50 \%$ in nerves, which have to be stretched to an elongation of $11 \%$. The flow is reduced as much as $100 \%$ with a strain of $15.7 \%$ (Topp and Boyd, 2006).

\section{Capsule-Like Structure in Cases of Brain Edema}

Just like the whole brain, circumscribed parts of the CNS are coated by capsule-like structures. Known examples include the pituitary gland, but also the optic nerve and the eyeball. These locations are completely or partially enclosed by either the dura mater or the sclera, respectively. The partial necrosis of the pregnancy induced hyperplastic pituitary gland as a complication of severe uterine bleeding during birth (Sheehan-syndrome) is caused by a local increase of tissue pressure due to edema in the fossa hypophysialis. The retinal degeneration in glaucoma is also a known example of pressure induced pathology in an encapsulated space. Neuromyelitis optica (Devic's syndrome) and idiopathic intracranial hypertension, but also MS are demyelinating diseases often including the optic nerve that is surrounded by the dura mater. In the knowledge of the hazardous tissue pressure in idiopathic intracranial hypertension, the surgical procedures of optic nerve decompression are carried out in order to prevent blindness (Acheson, 2006).

\section{Increased Pressure at the Edge of the Ventricles and the Deep White Matter}

An enlargement of the brain by edema reduces the fluid area in the subarachnoidal space, the basal cisterns and the ventricles.

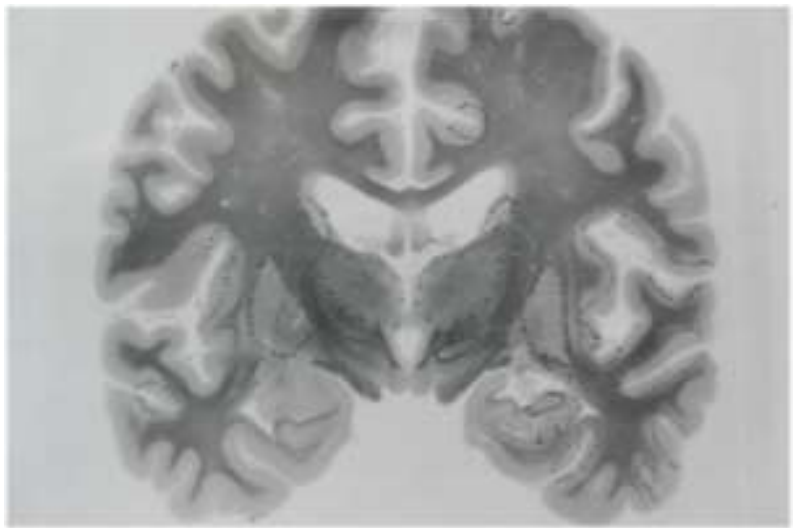

Fig. 3. Death of a 57-year old woman in diabetic coma. Hypoxemic necrosis of nerve cells in areas of elevated brain tissue pressure. The lesions are seen especially in the depth of the sulci, the gyri of the insula and the subcortical gray matter

The compression forces result in deformation or displacement of the white matter, especially at the rim of the shrunken ventricles. This pressure phenomenon can be compared to the compression at the inner concave side of an eraser when it is bent.

In prematurity or birth with perinatal complications, infants are often seen with periventricular localized white matter necrosis, called periventricular leucomalacia. This characteristic necrosis is caused by prolonged asphyxia and hypoxia with edema and circulatory disturbances (Rorke, 1992).

It is well-known that MS lesions are often observed at the lateral angle of the lateral ventricles. Moreover, lesions around the subependymal veins are observed at the edge of all ventricles and the aquaeductus cerebri (Allen and Kirk, 1992). Large periventricular demyelinated lesions of both cerebral hemispheres, such as seen in Schilder's disease (a special form of MS), mainly occur in young patients (Allen and Kirk, 1992). The increased pressure in the periventricular white matter, resulting in inadequate blood perfusion, may explain the formation of the spatial periventricular demyelinated lesions in disorders of the white matter like Binswanger's encephalopathy and leukoaraiosis (Baust et al., 1963; Beggs, 2013). Arteriosclerosis, arterial hypertension and every general brain swelling (e.g., edema in cases of uremia or blood pressure crisis) act synergistically with demyelinating processes. The deep white matter is particularly vulnerable to microangiopathy and chronic hypoperfusion because the nourishing blood vessels have the longest course of all penetrating brain vessels (Brown et al., 2009).

Typical for MS and leukoaraiosis in the periventricular region is the occurrence of venous vessels with thickened, hyalinized walls. This picture is known as periventricular venous collagenosis (Allen and Kirk, 
1992; Moody et al., 1995). This characteristic change of the venous wall is most likely induced by the reduced blood flow and hypoxemia. Collagenosis is not a primary vessel disease.

\section{Reduced Reabsorption of CSF and Brain Tissue Pressure}

There is some evidence that a reduced reabsorption of the CSF at the level of the cranial and spinal nerves causes an increase in pressure in the CSF-spaces and the brain tissue. An example is the hydrocephalus maleresorptivus often caused by obstruction of the drainage pathways following subarachnoid hemorrhage or purulent meningitis. The morbid obesity and the excess of body weight in cases of idiopathic intracranial hypertension is also a substantial factor in the pathogenesis of demyelinated lesions in the brain. The fat tissue coats the spinal nerves and an increase in fat tissue leads to an increased intraabdominal pressure (Binder et al., 2004). Both of these mechanisms may interfere with the reabsorption of the CSF into the lymphatic channels of the paravertebral nerves. Gastric surgery-induced weight loss for the treatment of the idiopathic intracranial hypertension is associated with a considerable reduction of the CSF-pressure, headaches and pulsatile tinnitus (Sugerman et al., 1999; Thambisetty et al., 2007).

CSF recruits not only from the choroid plexus but also to a large extent from the interstitial fluid of the brain. This interstitial fluid (comparable with the lymphatic fluid of other organs) originates from the exchange of fluids in the capillaries which takes place in all organs (Orescovic and Klarica, 2010; Bulat and Klarica, 2010). Despite the high pressure in the brain tissue in cases with idiopathic intracranial hypertension, the size of the brain ventricles is often not reduced. The constant metabolic formation of fluid by the brain tissue and the limited ability for the reabsorption of CSF may be the reason for that phenomenon (Miller and Adams, 1992; Jacobson et al., 1990).

\section{Discussion}

Various diseases and different factors may generate demyelination and necrosis of brain tissue. Infectious, toxic, traumatic, hereditary, degenerative and immunological causes are not discussed in this study. MS as one of the most prevalent demyelinating diseases has been included as it cannot be ruled out that edema or tissue pressure have an influence on the formation of MS lesions. This also applies to the other included demyelinating diseases of unknown etiology.

The reported examples above prove that an increased tissue pressure caused by edema may act in brain demyelination and tissue necrosis. In seldom cases, primary vascular diseases may also lead to tissue edema.
The hereditary angioedema is a genetic vascular disease that shows among other locations brain edema with demyelinated lesions. The disease is accompanied with often reversible symptoms like headache, pareses, ophthalmoplegic migraine and amaurosis (Krause et al., 1979).

Irrespective of the unknown etiology for MS the question arises as to why this pathodynamic principle is particularly apparent in young women. The answer is hypothetical. A hormonal influence appears to be possible.

Edema of allergic, toxic or inflammatory nature (e.g., flu like infections) and any feeling of discomfort accompanied by headache could be a trigger for MS attacks in men and women. However, fluid retention in the premenstrual syndrome of up to $1.5-41$ in $30-70 \%$ of women (Goeretzlehner et al., 2011) and the pregnancyor hormone-related tendency to accumulate fluid in the whole body may be a factor in the increased occurrence of brain edema, menstrual migraine and demyelinating diseases in young women. It is known that headache is often a symptom of brain swelling by edema. Tension or migraine-like headaches especially affect females. A comprehensive meta-analysis shows a significant association between migraines and MS. MS patients report migraines twice as often as controls (Pakpoor et al., 2012).

\section{Conclusion}

There is clear evidence that the lesions in many demyelinating diseases are caused by a local reduction in the blood circulation. However, this occurs seldomly due to cardiac insufficiency or blood vessel disorders but may be due to an increase in brain tissue pressure often caused by edema and consecutive tensile and compressive forces by stretched swollen gray and white matter. A capsule-like coating of enclosed edematous tissue areas may lead to a localized increase in tissue pressure. Another pathodynamic principle that acts in an increased brain tissue pressure can be the impaired reabsorption of CSF. All these phenomena may lead to a decreased blood circulation in the whole intracranial space or in different regions of the brain and may generate consecutively partial or complete demyelinated lesions or tissue necrosis. An improvement in the symptoms and a decrease of acute dysfunction is very likely after the reabsorption of the edema of acute demyelinating lesions. The immediate anti-edematous effect of high-dose corticosteroids may be a reason for the efficacious corticosteroid treatment of acute MS attacks.

Ischemia caused by edema or an elevated tissue pressure is not unknown, however, the knowledge of the effectiveness of this mechanism is still incomplete. Further research by pathologists, neurologists and neurophysiologists will be useful for the understanding 
of an increased tissue pressure in the pathogenesis of a more or less extensive part of demyelinating diseases.

\section{Acknowledgement}

The authors thank Dr. Ewan Scott and Dr. habil. Wibke Baumgarten for their critical review of the manuscript. Another word of thanks goes to Kristen McLean and Sabine Jones for their help to translate the text from German into English.

\section{Funding Information}

This work has not been supported by any institution. There are no financial or other relationships that might lead to a perceived conflict of interest.

\section{Author's Contributions}

All authors have made substantial contributions to the following:

Conception and design of the pressure hypothesis, the acquisition and interpretation of data; drafting the article and revising it critically for intellectual content. All authors have read the finished manuscript and approve of its publication in the present form.

\section{References}

Acheson, J.F., 2006. Idiopathic intracranial hypertension and visual function. Br. Med. Bull., 79-80: 233-244. PMID: 17242038

Allen, I.V. and J. Kirk, 1992. Demyelinating Diseases. In: Greenfield's Neuropathology, Adams, J.H. and L.W. Duchen, (Eds)., Edward Arnold, London, ISBN-10: 0195209486, pp: 447-520.

Baust, W., H. Niemczyk and H. Schäfer, 1963. Die beeinflussung des liquordruckes durch akute hämodynamische veränderungen. Z. Ges. Exp. Med., 136: 619-629. DOI: 10.1007/BF02053631

Beggs, C.B., 2013. Venous hemodynamics in neurological disorders: An analytical review with hydrodynamic analysis. BMC Med., 11: 142-142. DOI: $10.1186 / 1741-7015-11-142$

Binder, D.K., J.C. Horton, M.T. Lawton and M.D. McDermott, 2004. Idiopathic intracranial hypertension. Neurosurgery, 54: 538-552.

Brown, W.R., D.M. Moody, C.R. Thore, J.A. Anstrom and V.R. Challa, 2009. Microvascular changes in the white mater in dementia. J. Neurol. Sci., 283: 28-31. DOI: 10.1016/j.jns.2009.02.328.

Bulat, M. and M. Klarica, 2010. Recent insights into a new hydrodynamics of the cerebrospinal fluid. Brain Res. Rev., 65: 99-112. PMID: 20817024

Chua, G.C., Y.Y. Sitoh, C.C. Lim, H.C. Chua and P.Y. $\mathrm{Ng}$, 2002. MRI findings in osmotic myelinolysis. Clin. Radiol., 57: 800-806. PMID: 12384105
Ge, Y., V.M. Zohrabian, E.O. Osa, J. Xu and H. Jaggi et al., 2009. Diminished visibility of cerebral venous vasculature in multiple sclerosis by susceptibilityweighted imaging at 3.0 T. J. Magn. Reson. Imaging, 29: 1190-1194. PMID: 19388109

Goeretzlehner, G., C. Lauritzen, T. Roemer and W. Rossmanith, 2011. Practical hormone therapy in gynecology. De Gruiter, Berlin.

Graham, D.I., 1992. Hypoxia and Vascular Disorders. In: Greenfield's Neuropathology, Adams J.H. and L.W. Duchen, (Eds.), Edward Arnold, London, ISBN-10: 0195209486, pp: 153-268.

Jacobson, D.M., P.N. Karanjia, K.A. Olson and J.J. Warner, 1990. Computed tomography ventricular size has no predictive value in diagnosing pseudotumor cerebri. Neurology, 40: 1454-1455. PMID: 2392235

Juurlink, B.H.J., 2013. The evidence for hypoperfusion as a factor in multiple sclerosis lesion development. Mult. Scler. Int., DOI: 10.1155/2013/598093

Kaster, T., I. Sack and A. Samani, 2011. Measurement of the hyperelastic properties of ex vivo brain tissue slices. J. Biomech., 44: 1158-1163. PMID: 21329927

Konofagou, E.E., T.P. Harrigan, J. Ophir and T.A. Krouskop, 2001. Poroelastography: Imaging the poroelastic properties of tissues. Ultrasound Med. Biol., 27: 1387-1397. PMID: 11731052

Krause, K.H., U. Rentrop and U. Mehregan, 1979. Cerebral manifestations in angioneurotic edema (author's transl). J. Neurol. Sci., 42: 429-435. PMID: 512675

Krug, H. and K.R. Sandig, 1965. Die gehirnkonsistenz bei diabetes mellitus und urämie. Virchows Arch. Path. Anat., 339: 1-9. DOI: 10.1007/BF00962809

Law, M., A.M. Saindane, Y. Ge, J.S. Babb and G. Johnson et al., 2004. Microvascular abnormality in relapsing-remitting multiple sclerosis: Perfusion MR imaging findings in normal-appearing white matter. Radiology, 231: 645-652. PMID: 15163806

Mancini, M., V.B. Morra, O. Di Donato, V. Maglio, R. Lanzillo and R. Liuzzi et al., 2012. Multiple sclerosis: Cerebral circulation time. Radiology, 262: 947-955. PMID: 22357894

Martin, R.J., 2004. Central pontine and extrapontine myelinolysis: The osmotic demyelination syndromes. J. Neurol Neurosurg Psychiatry, 75: 22 28. DOI: 10.1136/jnnp.2004.045906

Messert, B., W.W. Orrison, M.J. Hawkins and C.E. Quaglieri, 1979. Central pontine myelinolysis. Considerations on etiology, diagnosis and treatment. Neurology, 29: 147-160. PMID: 571058

Miller, D.H. and S.M. Leary, 2007. Primary-progressive multiple sclerosis. Lancet Neurol., 6: 903-912. DOI: $10.1016 / \mathrm{S} 1474-4422(07) 70243-0$ 
Miller, J.D. and J.H. Adams, 1992. The Pathophysiology of Raised Intracranial Pressure. In: Greenfield's Neuropathology, Adams, J.H. and L.W. Duchen, (Eds.), Edward Arnold, London, ISBN-10: 0195209486, pp: 69-105.

Moody, D.M., W.R. Brown, V.A. Challa and R.L. Anderson, 1995. Periventricular venous collagenosis: Association with leukoaraiosis. Radiology, 194: 469-476. PMID: 7824728

Orescovic, D. and M. Klarica, 2010. The formation of cerebrospinal fluid: Nearly a hundred years of interpretations and misinterpretations. Brain Res. Rev., 64: 241-262. PMID: 20435061

Pakpoor, J., A.E. Handel, G. Giovannoni, R. Dobson and S.V. Ramagopalan, 2012. Meta-analysis of the relationship between multiple sclerosis and migraine. PLOS One, 7: e45295-e45295. PMID: 23024814

Rorke, L.B., 1992. Perinatal Brain Damage. In: Greenfield's Neuropathology, Adams, J.H. and L.W. Duchen, (Eds.), Edward Arnold, London, ISBN-10: 0195209486, pp: 639-708.

Sugerman, H.J., W.L. Felton, A. Sismanis, J.M. Kellum and E.J. DeMaria et al., 1999. Gastric surgery for pseudotumor cerebri associated with severe obesity. Ann. Surg., 229: 634-640. PMID: 10235521.

Tatewaki, Y., K. Kato, Y. Tanabe and S. Takahashi, 2012. MRI findings of corticosubcortical lesions in osmotic myelinolysis: Report of two cases. Br. J. Radiol., 85: e87-e90. DOI: 10.1259/bjr/26842276
Thambisetty, M., P.J. Lavin, N.J. Newman and V. Biousse, 2007. Fulminant idiopathic intracranial hypertension. Neurology, 68: 229-232. DOI: 10.1001/jamaneurol.2013.1951

Topp, K.S. and B.S. Boyd, 2006. Structure and biomechanics of peripheral nerves: Nerve responses to physical stresses and implications for physical therapist practice. Phys. Ther., 86: 92-109. PMID: 16386065

Valdueza, J.M., F. Doepp, S.J. Schreiber, B.W. van Osten and K. Schmierer et al., 2013. What went wrong? The flawed concept of cerebrospinal venous insufficiency. J. Cereb. Blood Flow Metab., 33: 657-668. PMID: 23443168

Van Essen, D.C., 1997. A tension-based theory of morphogenesis and compact wiring in the central nervous system. Nature, 385: 313-318. PMID: 9002514

Zamboni, P., R. Galeotti, E. Menegatti, A.M. Malagoni and G. Tacconi et al., 2009. Chronic cerebrospinal venous insufficiency in patients with multiple sclerosis. J. Neurol. Neurosurg. Psychiatry, 80: 392-399. PMID: 19060024

Zivadinov, R., G.U. Poloni, K. Marr, C.V. Schirda and C.R. Magnano et al., 2011. Decreased brain venous vasculature visibility on susceptibility-weighted imaging venography in patients with multiple sclerosis is related to chronic cerebrospinal venous insufficiency. BMC Neurol., 11: 128-128.

PMID: 22011402 